\title{
The Pervasive Role of the Nuclear Symmetry Energy in the Structure and Evolution of Neutron Stars
}

\author{
M. Prakash* \\ Department of Physics and Astronomy, Ohio University \\ Athens, Ohio 45701-2979, USA \\ E-mail: prakash@harsha.phy.ohiou.edu
}

\section{PALS}

The multifaceted role of the density dependent nuclear symmetry energy in the nuclear astrophysics involving neutron stars is highlighted. Efforts toward a model independent determination of the dense matter equation state through a deconstruction of the neutron star structure equation utilizing the masses and radii of several individual neutron stars are described. The need for observational data of both measurements for the same star is stressed.

10th Symposium on Nuclei in the Cosmos

July 27 - August 12008

Mackinac Island, Michigan, USA

Speaker.

$\dagger$ MP thanks his "PALS" Paul Ellis (in memoriam), James Lattimer, Sergey Postnikov, and Andrew Steiner who have helped and contributed significantly to this talk. Research support of the US Department of Energy under grant DE-FG02-93ER40756 is gratefully acknowledged. 


\section{Scope of This Talk}

In this talk, I will

1. summarize some theoretical advances made in establishing correlations between observables of nuclei and neutron stars,

2. indicate how laboratory experiments, e.g., at JLab and Rare Isotope Accelerators (RIA's), can help to unravel the composition and structure of a neutron star,

3. point out how the observed masses $\left(M^{\prime} \mathrm{s}\right)$ and radii $\left(R^{\prime} \mathrm{s}\right)$ of several individual neutron stars can be used to construct the model independent equation of state (EOS) of dense matter (through an inversion process that I term as "Deconstructing a Neutron Star"), and

4. indicate the key neutron star observations that are necessary to take leaps in our understanding of the nature of strong interactions at supra-nuclear densities.

\section{The Pervasive Role of the Nuclear Symmetry Energy}

The nuclear (a)symmetry energy

$$
E_{s y m}(n, \delta)=\frac{1}{2 n} \frac{\partial^{2} \varepsilon(n, x)}{\partial \delta^{2}}, \quad \delta=\frac{n_{n}-n_{p}}{n=n_{p}+n_{n}}=1-2 x,
$$

represents the energy cost required to create an isospin asymmetry $\delta$ in nucleonic matter. Above, $n_{n}$ and $n_{p}$ are the neutron and proton densities, $x$ is the proton fraction in matter, and $\varepsilon(n, \delta)$ is the energy density of isospin asymmetric matter. Figure 1 from Ref. [1] shows the energy per particle of bulk nuclear $(x=1 / 2)$ and neutron matter $(x=0)$ versus the total baryon density $n$.

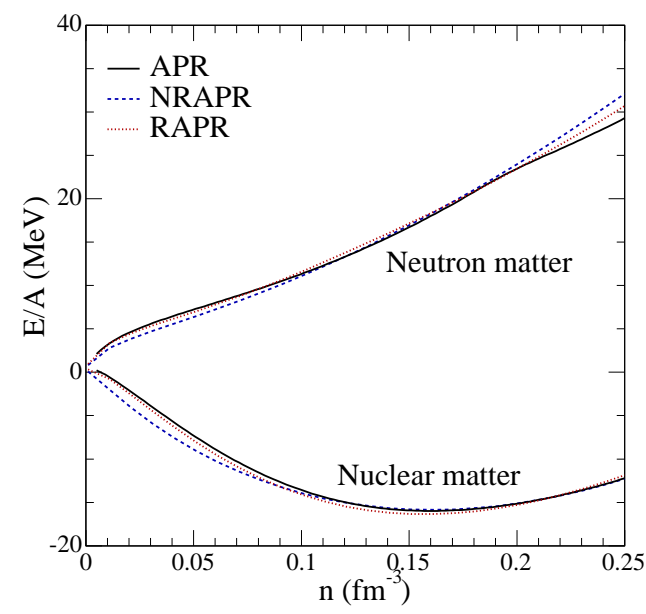

Figure 1: Results shown are for the microscopic potential model calculations of Akmal and Pandharipande (APR) [2,3] and simpler nonrelativistic and relativistic effective model fits (NRAPR and RAPR) [1]. 
The physical properties of nuclei, such as their masses, neutron and proton density distributions (including their mean radii), collective excitations, fission properties, matter and momentum flows in high energy heavy-ion collisions, etc. all depend on the isospin structure of the strong interactions between nucleons (i.e., $n n$ and $p p$ interactions versus $n p$ interactions). The energy $\hat{\mu}=\mu_{n}-\mu_{p} \cong 4 E_{s y m} \delta$, where $\mu_{n}$ and $\mu_{p}$ are the neutron and proton chemical potentials, respectively, is crucial in determining reaction rates involving electrons and neutrinos, particle abundances, etc., in astrophysical contexts such as supernova dynamics, proto-neutron star evolution, the $r$-process, the long-term cooling of neutron stars, and the structure of cold-catalyzed neutron stars (i.e. their masses, radii and crustal extent), etc. The pervasive role of the isospin dependence of strong interactions in nuclear processes in the laboratory and the cosmos is sketched in Fig. 2.

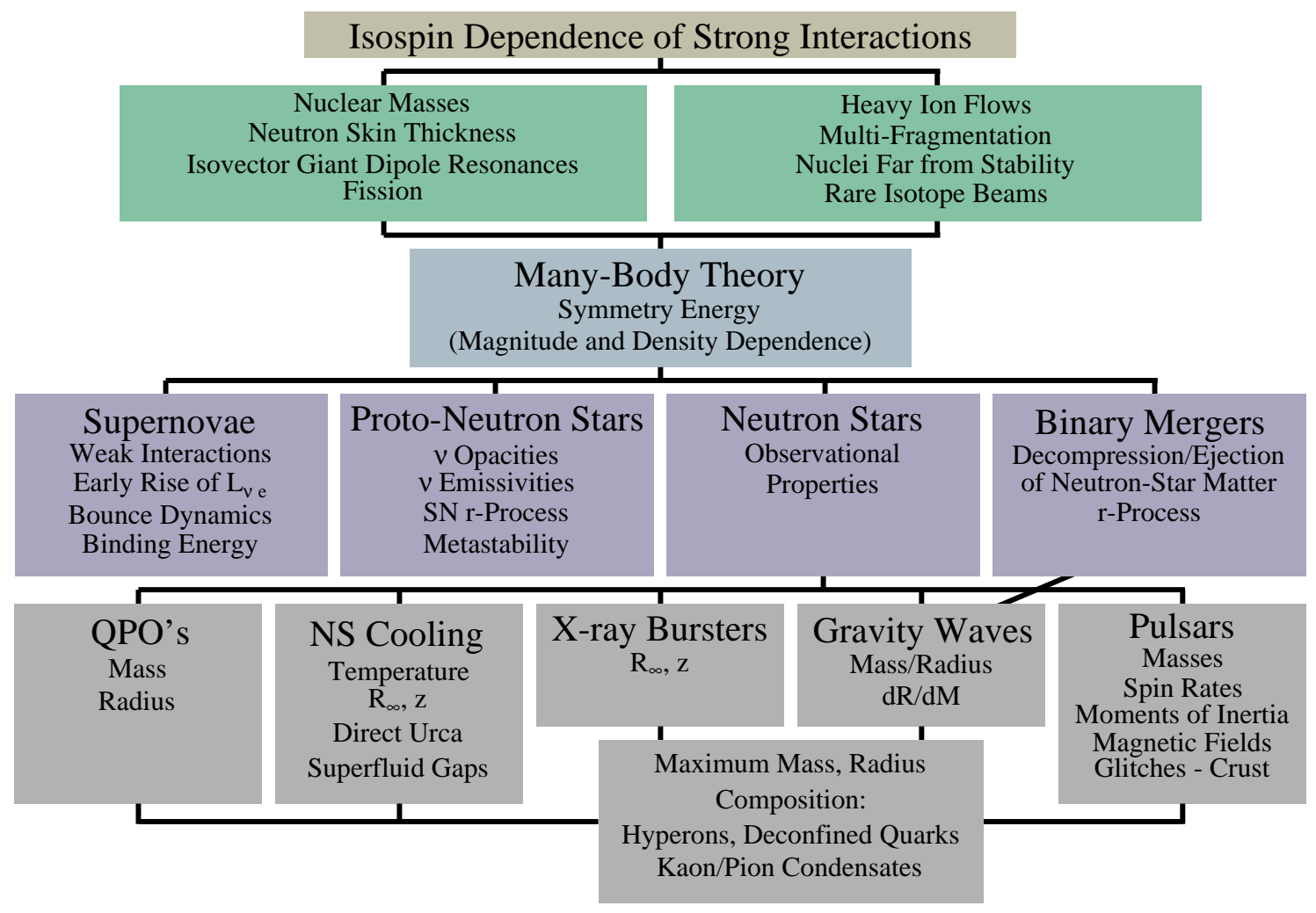

Figure 2: The pervasive role of the nuclear symmetry energy. Figure taken from Ref. [1].

\section{Connections to Neutron Star Structure}

The structure of neutron stars is determined by the energy and pressure of charge-neutral betastable matter, the nucleonic components of which can be written as

$$
\begin{aligned}
& E(n, x) \cong E(n, 0.5)+E_{\text {sym }}(n)(1-2 x)^{2}+\cdots, \\
& P(n, x) \cong n^{2}\left[E^{\prime}(n, 0.5)+E_{\text {sym }}^{\prime}(1-2 x)^{2}\right]+\cdots,
\end{aligned}
$$


where the primes above refer to derivatives with respect to density. Leptonic (electrons and muons) contributions must be added to Eq. (3.1) for the total energy and pressure. As demonstrated in Ref. [4], the radius (but, not necessarily the mass) of a nucleonic neutron star is chiefly determined by the pressure in the vicinity of the nuclear equilibrium density, $n_{0} \simeq 0.16 \pm 0.01 \mathrm{fm}^{-3}$, for which the contribution of the first term to the pressure in Eq. (3.1) is very small. This feature of a neutron star's structure highlights the importance of the density dependence of the nuclear symmetry energy near $n_{0}$. Figure 3 shows the mass-radius relationships for select EOS models including the cases in which extreme softening due to the presence of exotica such as hyperons, quarks or Bose condensates occurs in the cores of stars.

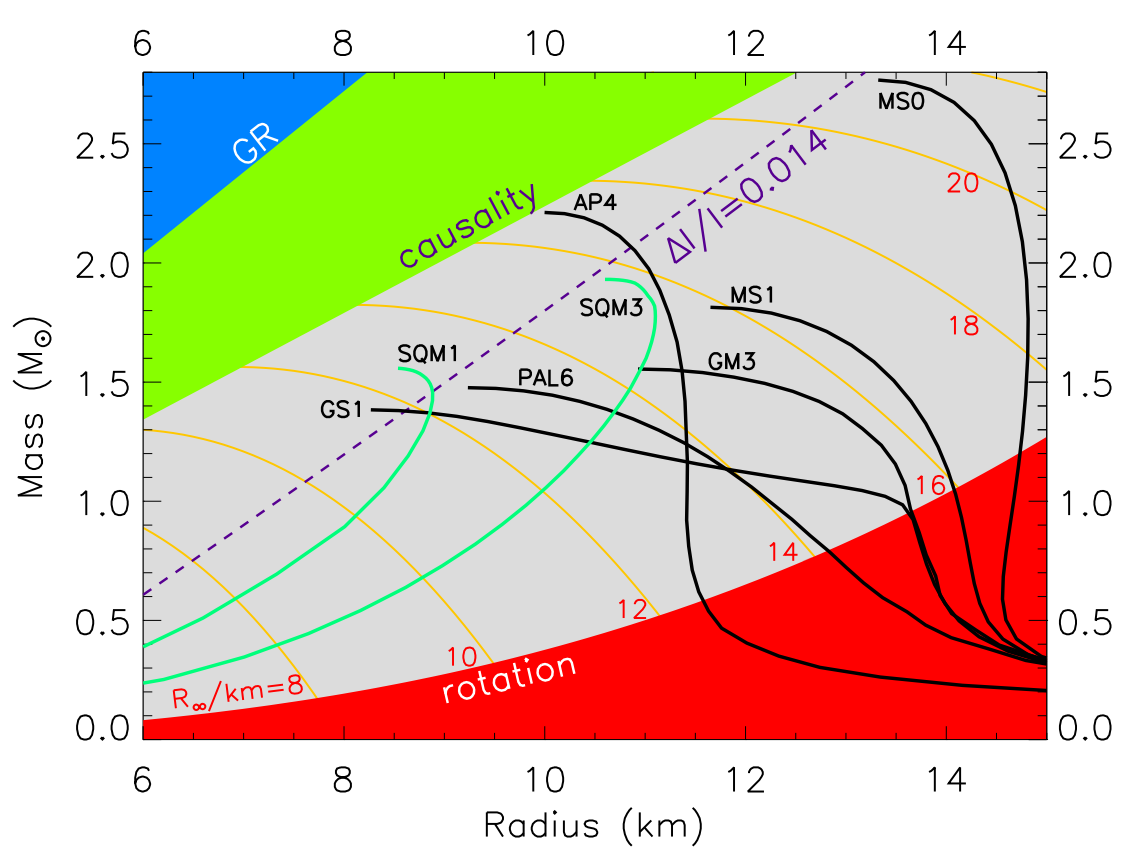

Figure 3: Mass versus radius curves from Ref. [5]. EOS symbols are as in Ref. [4].

In Fig. 4, the observed neutron star masses are shown. The simple and error weighted means for neutron stars in x-ray binaries are $1.55 \mathrm{M}_{\odot}$ and $1.37 \mathrm{M}_{\odot}$, respectively. The corresponding numbers for double neutron star binaries are $1.32 \mathrm{M}_{\odot}$ and $1.41 \mathrm{M}_{\odot}$, whereas for white dwarf neutron star binaries, the numbers are $1.60 \mathrm{M}_{\odot}$ and $1.33 \mathrm{M}_{\odot}$. It is unfortunate that in those cases (e.g., binary radio pulsars) in which the masses are very accurately known, the corresponding numbers for radii are unknown. It is hoped that with improvements in timing analysis, the moment of inertia of the neutron star in the double-pulsar system J0737-3039 will become available [6]. As will be shown later, the masses and radii of several individual neutron stars can uniquely pin down the EOS of dense matter. For prospects of obtaining this much needed information, see the article by Bob Rutledge in these proceedings. For prognosis of EOS determination, see Ref. [7]. 


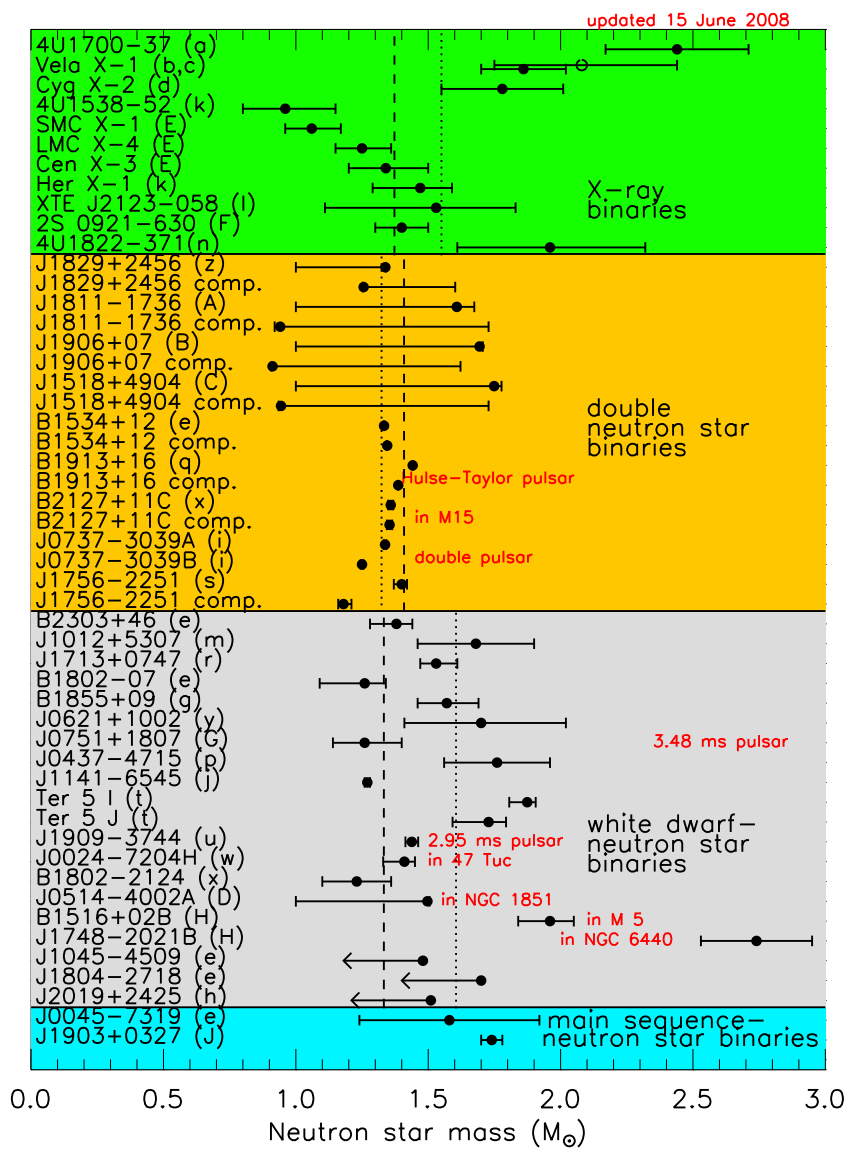

Figure 4: Observed neutron star masses. Figure courtesy of Lattimer.

\section{The Role of Isospin Interactions in Nuclei and Neutron Stars}

A few of the recently discovered empirical relationships that underscore the role of isospin interactions in nuclei and neutron stars are highlighted here.

\section{The neutron skin thickness in nuclei and the sub-nuclear pressure of pure neutron matter}

Typel and Brown $[8,9]$ have noted that model calculations of the difference between neutron and proton rms radii $\delta R=\left\langle r_{n}^{2}\right\rangle^{1 / 2}-\left\langle r_{p}^{2}\right\rangle^{1 / 2}$ are linearly correlated with the pressure of pure neutron matter at a density below $n_{0}$ characteristic of the mean density in the nuclear surface (e.g., $\left.0.1 \mathrm{fm}^{-3}\right)$. The density dependence of the symmetry energy controls the so-called neutron skin thickness $\delta R$ in a heavy, neutron-rich nucleus. Explicitly, $\delta R$ is proportional to a specific average of $\left[1-E_{\text {sym }}\left(n_{0}\right) / E_{\text {sym }}(n)\right]$ in the nuclear surface, see Refs. [10,1].

The left panel of Fig. 5 shows the Typel-Brown correlation extended to neutron-star matter in Ref. [1] using both potential and field-theoretical models. The proposal [11, 12] at JLab to measure the neutron rms radius of ${ }^{208} \mathrm{~Pb}$ through parity-violating electron scattering experiments to $1 \%$ accuracy (the rms charge radii of nuclei are known to better accuracy) can help to provide a 

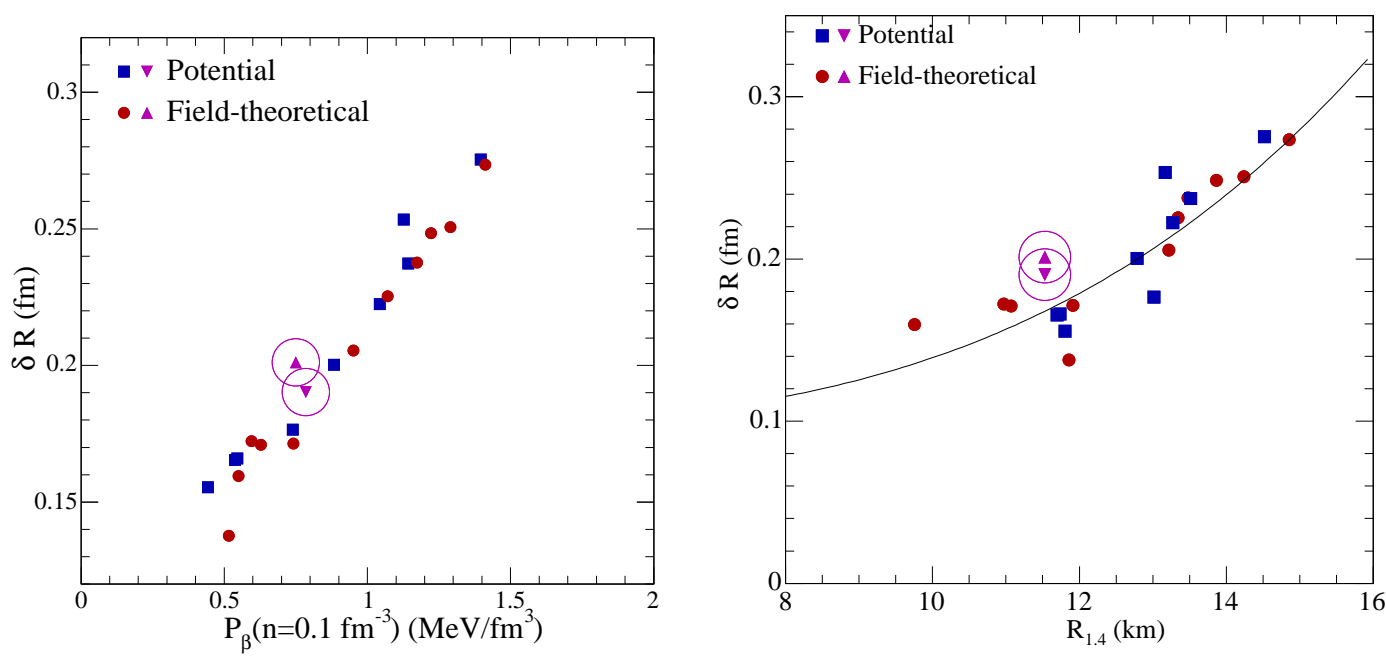

Figure 5: Left panel: The neutron skin thickness $\delta R$ of finite nuclei versus the pressure of $\beta$-equilibrated neutron star matter at a density of $0.1 \mathrm{fm}^{-3}$. Right panel: Calculated neutron skin thicknesses $\delta R$ of nuclei versus the radii of $1.4 \mathrm{M}_{\odot}$ stars. Trends for stars up to the maximum mass are similar. The solid lines represents a least square fit. Trends for stars up to the maximum mass are similar [1]. Figure taken from [1].

calibration pressure of neutron-star matter at sub-nuclear densities. The knowledge of neutron rms radii for neutron-rich nuclei of varying masses will be of great help in establishing the uncertain density dependence of isospin interactions at sub-nuclear densities. The right panel of Fig. 5 shows that the correlation between $\delta R$ and $R_{1.4}$ is not very sharp. Accurate predictions of neutron star radii require knowledge of the equation of state at supra-nuclear densities.

\section{The neutron skin thickness in nuclei and the neutron star radius}

Horowitz and Piekarewicz [13] have pointed out that models that yield smaller neutron skins in heavy nuclei tend to yield smaller neutron star radii. These authors, along with others [14, 1], have stressed the need for accurate measurements of the neutron skin thicknesses of nuclei. Although the neutron star radii are determined at supra-nuclear densities, the calibration provided at subnuclear densities cannot be ignored. Even with varying stiffness at high density, several works have confirmed the trend shown in the right panel of Fig. 5. The Horowitz-Piekarewicz correlation is thus very suggestive inasmuch as radii of stars up to the maximum mass exhibit similar trends. It would be fruitful to test this correlation from accurate radius measurements of neutron stars whose corresponding masses are also well determined.

\section{The neutron star radius $R$ and the pressure $P$ of neutron-star matter}

Lattimer and Prakash [4] found that the quantity $R P^{-1 / 4}$ is approximately constant, for a given neutron star mass, for a wide variety of EOS's when the pressure $P$ of beta-equilibrated neutronstar matter is evaluated at a density in the range $n_{0}$ to $2 n_{0}$, where $n_{0}$ denotes equilibrium nuclear matter density. Since the pressure of nearly pure neutron matter (a good approximation to neutron 
star matter) near $n_{0}$ is approximately given by $n^{2} E_{s y m}^{\prime}$ (see Eq. (3.1)), the density dependence of the symmetry energy just above $n_{0}$ will be a critical factor in determining the neutron star radius. Figure 6 shows results for $1.4 M_{\odot}$ stars. Similar trends persist for stars up to the maximum mass star as shown in Ref. [4]. This correlation, coupled with accurate measurements of neutron star radii, can delimit the very uncertain (up to a factor of 5!) pressure of neutron-star matter (see Ref. [4]) at supra-nuclear densities.

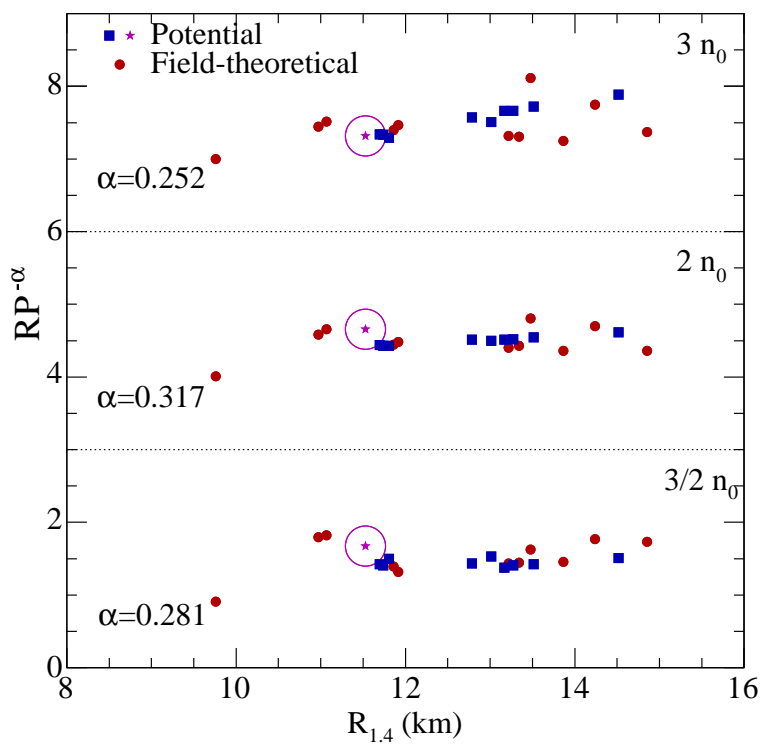

Figure 6: The quantity $R P^{-\alpha}$ as a function of the stellar radius $R$, for pressures $P$ determined at 3/2, 2 and 3 times equilibrium nuclear matter density. For each density, the best-fit value for the exponent $\alpha$ is as indicated. Circled stars indicate the results obtained with the APR equation of state. These results are for $1.4 \mathrm{M}_{\odot}$ stars; results for stars up to the maximum mass star are similar. Figure taken from Ref. [1].

\section{Deconstructing a Neutron Star}

This section contains a report of on-going work of the speaker with Sergey Postnikov and James Lattimer [15]. The question being posed here is "Can observations of the masses and radii of several (say 5 to 7) individual stars uniquely determine the dense matter EOS?" In short, the answer is a resounding YES! This assertion follows from the work of Lindblom [16] who exploited the one-to-one correspondence between an EOS and the $M-R$ curve generated through the use of the Tolman-Oppenheimer-Volkov (TOV) [17, 18] equations of stellar structure. Given the diverse theoretical predictions, it is of great interest to enquire whether or not observations of masses and radii of several neutron stars can be used to uniquely pin down the poorly known high density EOS. The extent to which inherent errors in measurements affect the extraction of the EOS (not addressed in Lindblom's work) will become an important issue.

Recently launched campaigns [19] to accurately measure masses and radii of a large number of neutron stars give impetus to investigate this inversion procedure in more depth than originally 
undertaken by Lindblom. The extension of such an inversion procedure to include other observables such as the surface redshifts, binding energies, moments of inertia, etc. has been undertaken by Postnikov, Prakash and Lattimer incorporating the inherent errors involved in observations.

We begin by recasting the TOV equations of stellar structure using the variable $h$ defined through $d h=d p /(p+\rho(p))$, where $p$ is the pressure and $\rho(p)$ is the mass-energy density. Adopting the units $G=1$ and $c=1$, the result is

$$
\frac{d r^{2}}{d h}=-2 r^{2} \frac{r-2 m}{m+4 \pi r^{3} p}, \quad \frac{d m}{d h}=-4 \pi r^{3} \rho \frac{r-2 m}{m+4 \pi r^{3} p},
$$

where $p(h)$ and $\rho(h)$, which serve as input, contain the EOS. The advantages of this reformulation (somewhat different than that used by Lindblom) are that the enclosed mass $m$ and radius $r$ are now dependent (on $h$ and thus the EOS) variables, and $h$ is finite both at the center and surface of the star. Furthermore, the quantities $r^{2}(h)$ and $m(h)$ admit tractable Taylor expansions about $h_{c}$ that feature the finite quatities $p_{c}, \rho_{c}$ and $(d \rho / d h)_{c}$, where the subscript $c$ denotes the star's center.

The procedure is to begin with a known EOS up to a certain density, take small increments in mass and radius, and adopt an iterative scheme based on Eq. (5.1) to reach the new known mass and radius. In the absence of observational numbers for individual stars, a test of the above scheme can be performed by assuming that the masses and radii are those that result from a model EOS.

We have tested our scheme using an EOS consisting of two polytropes whose indices are appropriately chosen to mimic a realistic EOS and obtained results that reproduce the exact EOS to hundredths of percent accuracy. The left panel of Fig. 7 shows our results for this test case. Methods to yield even better accuracy using this modified Lindblom procedure are being devised.

An alternative procedure is to solve the reformulated TOV equations from the center to the surface with the assumed form of the EOS using a Newton-Raphson scheme to obtain the known mass and radius. The results from this approach is shown in the right panel of Fig. 7. This method also yields results to hundredths of percent accuracy. We now have two methods that yield similarly accurate results.

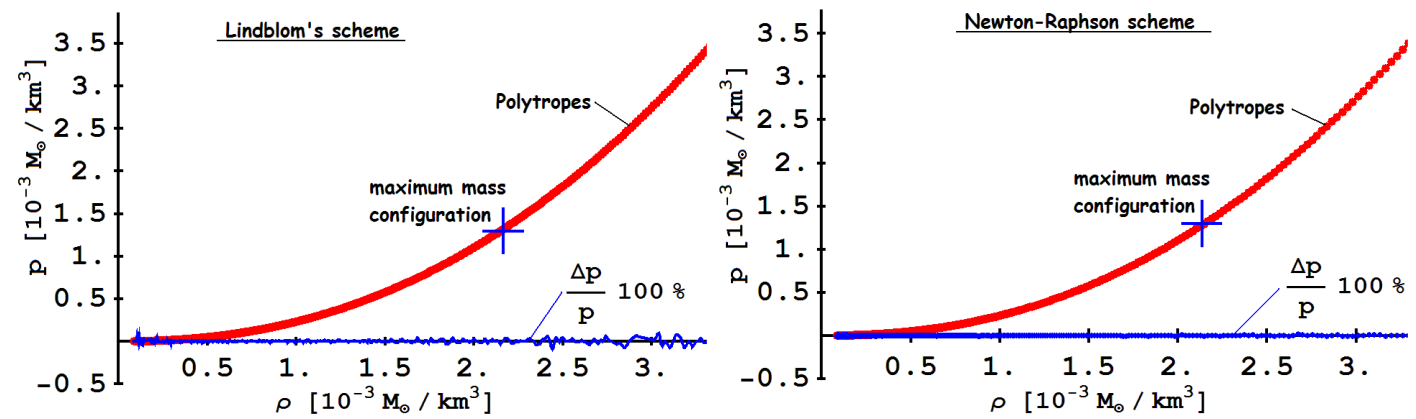

Figure 7: Deconstructing a neutron star with a two-power polytropic EOS.

We are now in a position to test our two schemes using physically motivated model EOS's. As examples of realistic EOS's, we will begin with the EOS's of Ref. [20] . These EOS's allow for variations in the uncertain high-density behavior of the nuclear symmetry energy and mimic trends found in microscopic calculations of high-density matter. The EOS labeled PAL31 is characterized 
by the nuclear matter compression modulus of $K=240 \mathrm{MeV}$ and a symmetry energy function for potential interactions: $F(u)=u$, where $u=n / n_{0}$ and $n_{0}=0.16 \mathrm{fm}^{-3}$. Including contributions from kinetic energy, the symmetry energy at $n_{0}$ is $30 \mathrm{MeV}$. The results in Fig. 8 show the extent to which the EOS is reconstructed for the case in which the masses and radii are assumed to be those that result from the EOS of PAL31 [20]. The two iterative schemes described above yield results of similar satisfactory accuracy.
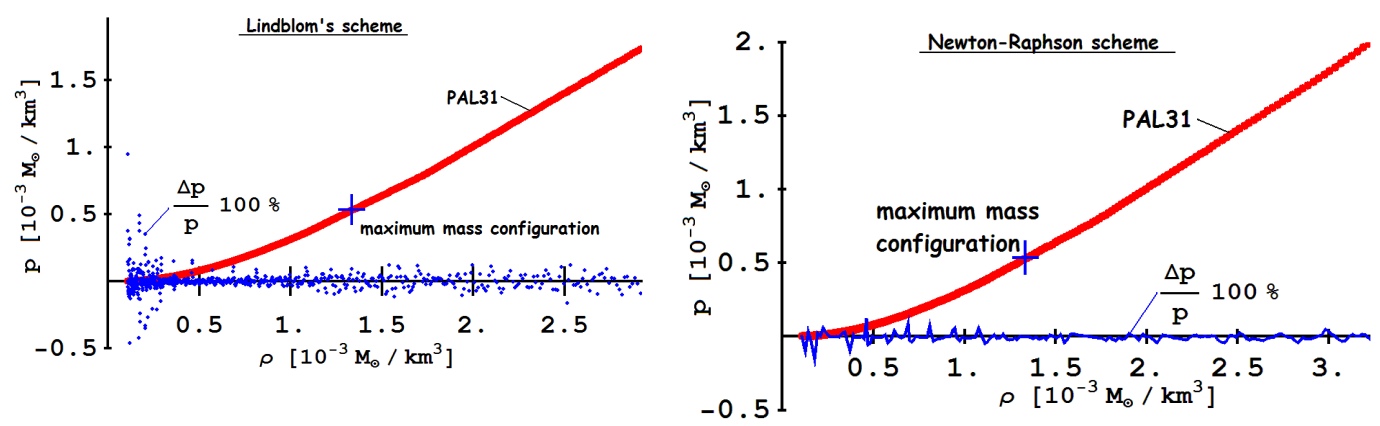

Figure 8: Deconstructing a neutron star with a physically motivated nucleonic EOS.

Work is in progress to determine the extent to which "Deconstruction" works accurately for a variety of EOS's including those that permit extreme softening at high density. Several exercises can be envisaged: (i) To what extent can one determine if only one mass and radius are very well determined? (ii) What if several masses and radii are known, albeit with unsatisfactory error bars? (iii) To what extent can we determine the EOS given the inherent error bars in measurements? Answers to these questions and many others will be published soon [15]. In addition to its inherent worth, this investigation will aid astronomers to better plan and secure observations in the future.

\section{Conclusions}

It is clear that

1. the masses and radii of several (say 5 to 7 ) of individual neutron stars can pin down (through deconstruction) the equation of state of neutron-star matter and shed light on the density dependence of the symmetry energy, i.e., the poorly known isospin dependence of strong interactions, and

2. precise laboratory experiments, particularly those involving neutron-rich nuclei, are sorely needed to pin down the near-nuclear aspects of the symmetry energy which determines the masses, neutron skin thicknesses, collective excitations, etc., of nuclei.

It is also clear that continuing mutual interactions of astronomers, laboratory experimenters and theorists in the fields of astrophysics and strong interaction physics are necessary to fulfill the objective of understanding the physics of compact objects in which the ultimate energy density of observable cold-baryonic matter is realized [21]. 


\section{References}

[1] A. W. Steiner, M. Prakash, J. M. Lattimer and P. J. Ellis, Phys. Rep. 411 (2005) 325.

[2] A. Akmal and V. R. Pandharipande, Phys. Rev. C 58 (1997) 2261.

[3] A. Akmal, V. R. Pandharipande and D. G. Ravenhall, Phys. Rev. C 65 (1998) 1804.

[4] J. M. Lattimer and M. Prakash, Astrophys. J1. 550 (2001) 426.

[5] J. M. Lattimer and M. Prakash, Science 304 (2004) 536.

[6] J. M. Lattimer and B. F. Schutz, Astrophys. J1. 629 (2005) 979.

[7] J. M. Lattimer and M. Prakash, Phys. Rep. 442 (2007) 109.

[8] B. A. Brown, Phys. Rev. Lett. 85 (2000) 5296.

[9] S. Typel and B. A. Brown, Phys. Rev. C 64 (2001) 027302.

[10] H. Krivine, J. de Phys. C 6 (Supp.) (1984) 153.

[11] C. J.Horowitz, S. J. Pollock, P. A. Souder, R. Michaels, Phys. Rev. C 63 (2001) 025501.

[12] R. Michaels, P. A. Souder, G. M. Urciuoli, Proposal PR-00-003, Jefferson Laboratory, 2000.

[13] C. J. Horowitz and J. Piekarewica, Phys. Rev. Lett. 86 (2001) 5647.

[14] R. J. Furnstahl, Nucl. Phys. A 706 (2002) 85.

[15] S. Postnikov, M. Prakash and J. M. Lattimer, In preparation.

[16] L. Lindblom, Astrophys. J1. 398 (1992) 569.

[17] R. C. Tolman, Proc. Nat. Acad. Sci., U. S. A., 20 (1934) 3.

[18] J. R. Oppenheimer and G. M. Volkoff, Phys. Rev. 55 (1939) 374.

[19] See the contribution from Rutledge in these proceedings.

[20] M. Prakash. T. L. Ainsworth and J. M. Lattimer, Phys. Rev. Lett. 61 (1988) 2518.

[21] J. M. Lattimer and M. Prakash, Phys. Rev. Lett. 94 (2005) 111101. 